\title{
Establishing the first hidden-charm pentaquark with strangeness
}

\author{
Hua-Xing Chen ${ }^{1, \mathrm{a}}$, Wei Chen ${ }^{2, \mathrm{~b}}$, Xiang Liu ${ }^{3,4, \mathrm{c}}$, Xiao-Hai Liu ${ }^{5, \mathrm{~d}}$ \\ ${ }^{1}$ School of Physics, Southeast University, Nanjing 210094, China \\ 2 School of Physics, Sun Yat-Sen University, Guangzhou 510275, China \\ ${ }^{3}$ School of Physical Science and Technology, Lanzhou University, Lanzhou 730000, China \\ ${ }^{4}$ Research Center for Hadron and CSR Physics, Lanzhou University and Institute of Modern Physics of CAS, Lanzhou 730000, China \\ ${ }^{5}$ Center for Joint Quantum Studies and Department of Physics, School of Science, Tianjin University, Tianjin 300350, China
}

\begin{abstract}
We study the $P_{c S}(4459)^{0}$ recently observed by LHCb using the method of QCD sum rules. Our results support its interpretation as the $\bar{D}^{*} \Xi_{c}$ hadronic molecular state of either $J^{P}=1 / 2^{-}$or $3 / 2^{-}$. Within the hadronic molecular picture, the three $\mathrm{LHCb}$ experiments observing $P_{c}$ and $P_{c s}$ states (Aaij et al., Phys Rev Lett 115:072001, 2015; Aaij et al., Phys Rev Lett 122:222001, 2019; Aaij et al., arXiv:2012.10380 [hep-ex], 2012) can be well understood as a whole. This strongly supports the existence of hadronic molecules, whose studies can significantly improve our understanding on the construction of the subatomic world. To verify this picture, we propose to further investigate the $P_{C s}(4459)^{0}$ to examine whether it can be separated into two states, and to search for the $\bar{D} \Xi_{c}$ molecular state of $J^{P}=1 / 2^{-}$.
\end{abstract}

\section{Introduction}

Atomic nuclei are made of protons and neutrons, which are themselves composed of quarks and gluons. In the past century a huge number of subatomic particles, called hadrons, were discovered in particle experiments, whose properties are similar to the proton and neutron [4]. One naturally raises an interesting question: are there subatomic particles corresponding to the nucleus? Nowadays we call them "hadronic molecules", whose studies can significantly improve our understanding on the construction of the subatomic world, as illustrated in Fig. 1.

It is not so easy to answer the above question. Most of the experimentally observed hadrons can be described

\footnotetext{
a e-mail: hxchen@buaa.edu.cn (corresponding author)

be-mail: chenwei29@mail.sysu.edu.cn

c e-mail: xiangliu@1zu.edu.cn

de-mail: xiaohai.liu@tju.edu.cn
}

as $q \bar{q}$ mesons or $q q q$ baryons in the conventional quark model, while there can also exist (compact) $q \bar{q} q \bar{q}$ tetraquarks and $q q q q \bar{q}$ pentaquarks $[5,6]$, etc. With the experimental progress on this issue over the past decade, dozens of $X Y Z$ charmonium-like states were reported, providing us good opportunities to identify exotic hidden-charm tetraquarks [4]. However, these states may be explained as hadronic molecules, while they may also be explained as compact tetraquarks that are still hadrons.

Fortunately, in the LHCb experiments performed in 2015 and 2019 [1,2], the famous hidden-charm pentaquark states, $P_{c}$ (4312), $P_{c}$ (4380), $P_{c}$ (4440), and $P_{c}$ (4457), were discovered. These four $P_{c}$ states contain at least five quarks $\bar{c} c u u d$, so they are perfect candidates of hidden-charm pentaquark states. Among them, the three narrow states $P_{c}(4312)$, $P_{c}(4440)$, and $P_{c}(4457)$ are just below the $\bar{D} \Sigma_{c}$ and $\bar{D}^{*} \Sigma_{c}$ thresholds, so their natural interpretations are the $\bar{D} \Sigma_{c}$ and $\bar{D}^{*} \Sigma_{c}$ hadronic molecular states [7-11], whose existence had been predicted in Refs. [12-16] before the LHCb experiment performed in 2015 [1]. However, there still exist other possible explanations [17-22], and we refer to the reviews [23-28] for detailed discussions.

It is natural to conjecture whether the hidden-charm pentaquark state with strangeness exists or not. Such a state is usually denoted as " $P_{c s}$ ", whose quark content is $\bar{c} c s q q$ $(q=u / d)$. There have been some but not many theoretical studies on it [29-37]. Especially, we proposed in Ref. [38] to search for the $P_{c s}$ in the $J / \psi \Lambda$ invariant mass spectrum of the $\Xi_{b}^{-} \rightarrow J / \psi K^{-} \Lambda$ decays. Besides, in Ref. [39] the authors studied the $P_{C S}$ using the chiral effective field theory, and calculated the mass of the $J^{P}=1 / 2^{-} \bar{D}^{*} \Xi_{c}$ molecular state to be $4456.9_{-3.3}^{+3.2} \mathrm{MeV}$. Note that both of these two references are based on the hadronic molecular picture.

Very recently, the LHCb Collaboration reported the evidence of a hidden-charm pentaquark state with strangeness, $P_{c s}(4459)^{0}$, in the $J / \psi \Lambda$ invariant mass spectrum of the 


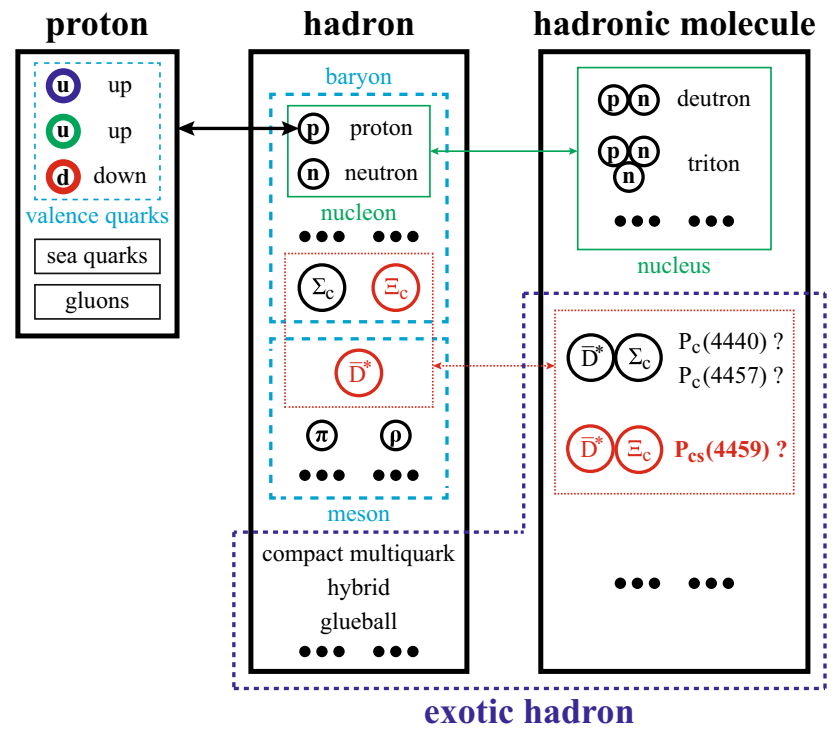

Fig. 1 A possible way to construct the subatomic world

$\Xi_{b}^{-} \rightarrow J / \psi K^{-} \Lambda$ decays [3]. Its mass and width were measured to be:

$$
\begin{aligned}
& P_{c s}(4459)^{0}: M=4458.8 \pm 2.9_{-1.1}^{+4.7} \mathrm{MeV}, \\
& \Gamma=17.3 \pm 6.5_{-5.7}^{+8.0} \mathrm{MeV},
\end{aligned}
$$

while its spin-parity quantum number was not determined since the statistic is not enough. Note that the channel observing the $P_{c s}(4459)^{0}$ is just the one proposed by us in Ref. [38], and the above mass value is almost identical to the mass of the $J^{P}=1 / 2^{-} \bar{D}^{*} \Xi_{c}$ molecular state predicted in Ref. [39]. Therefore, the present LHCb experiment [3] strongly supports the hadronic molecular picture once more.

Actually, as indicated by LHCb, the $P_{c s}(4459)^{0}$ is about $19 \mathrm{MeV}$ below the $\bar{D}^{* 0} \Xi_{c}^{0}$ threshold [3], so it is natural to interpret it as the $\bar{D}^{*} \Xi_{c}$ molecular state, with the spinparity quantum number $J^{P}=1 / 2^{-}$or $3 / 2^{-}$. Accordingly, in this letter we shall study the $\bar{D}^{*} \Xi_{c}$ molecular states of $J^{P}=1 / 2^{-}$and $3 / 2^{-}$using the method of QCD sum rules, and at the same time we shall also study the $\bar{D} \Xi_{c}$ molecular state of $J^{P}=1 / 2^{-}$. We calculate masses of $\bar{D}^{*} \Xi_{c}$ molecular states to be $4.46_{-0.14}^{+0.16} \mathrm{GeV}$ for the $J^{P}=1 / 2^{-}$ one and $4.47_{-0.15}^{+0.19} \mathrm{GeV}$ for the $J^{P}=3 / 2^{-}$one. These two values are both consistent with the experimental mass of the $P_{c s}(4459)^{0}$, supporting its interpretation as the $\bar{D}^{*} \Xi_{c}$ molecular state of either $J^{P}=1 / 2^{-}$or $3 / 2^{-}$. We also calculate the mass of the $J^{P}=1 / 2^{-} \bar{D} \Xi_{c}$ molecular state to be $4.29_{-0.12}^{+0.13} \mathrm{GeV}$.

To verify the hadronic molecular picture, we propose to further investigate the $P_{c S}(4459)^{0}$ state in future experiments to examine whether it can be separated into two states, and to search for the $J^{P}=1 / 2^{-} \bar{D} \Xi_{c}$ molecular state. If the hadronic molecular picture turns out to be correct, our under- standing on the construction of the subatomic world would be significantly improved. Besides, these studies are helpful to improve our understanding on the non-perturbative behaviors of the strong interaction at the low energy region.

\section{Hidden-charm pentaquark currents}

We use the $\bar{c}, c, s, u$, and $d$ quarks to construct hidden-charm pentaquark interpolating currents with strangeness. To study $\bar{D}^{(*)} \Xi_{c}$ molecular states, we consider the following type of currents:

$\eta(x)=\left[\bar{c}_{a}(x) \Gamma_{1} u_{b}(x)\right]\left[\left[d_{c}^{T}(x) \mathbb{C} \Gamma_{2} s_{d}(x)\right] \Gamma_{3} c_{e}(x)\right]$,

where $a \cdots e$ are color indices, $\Gamma_{1 / 2 / 3}$ are Dirac matrices, and $\mathbb{C}=i \gamma_{2} \gamma_{0}$ is the charge-conjugation operator. The other type of currents:

$\eta^{\prime}(x)=\left[\bar{c}_{a}(x) \Gamma_{1} d_{b}(x)\right]\left[\left[u_{c}^{T}(x) \mathbb{C} \Gamma_{2} s_{d}(x)\right] \Gamma_{3} c_{e}(x)\right]$,

can be similarly studied, but they just lead to the same QCD sum rule results as the $\eta(x)$ currents. Hence, the present study can not distinguish the isospin of $P_{c s}$ states.

The $\eta(x)$ currents can be constructed by combining charmed meson operators and charmed baryon fields. We need the charmed meson operators $J_{\mathcal{D}}$, which couple to the ground-state charmed mesons $\mathcal{D}=\bar{D}^{0} / \bar{D}^{* 0}$ :

$$
\begin{aligned}
J_{\bar{D}^{0}} & =\bar{c}_{d} \gamma_{5} u_{d}, \\
J_{\bar{D}^{* 0}} & =\bar{c}_{d} \gamma_{\mu} u_{d} .
\end{aligned}
$$

We also need the charmed baryon field $J_{\mathcal{B}}$, which couples to the ground-state charmed baryon $\mathcal{B}=\Xi_{c}^{0}$ :

$J_{\Xi_{c}^{0}}=\epsilon^{a b c}\left[d_{a}^{T} \mathbb{C} \gamma_{5} s_{b}\right] c_{c}$.

There can be altogether three $\bar{D}^{(*)} \Xi_{c}$ hadronic molecular states, that are $\bar{D} \Xi_{c}$ of $J^{P}=1 / 2^{-}, \bar{D}^{*} \Xi_{c}$ of $J^{P}=1 / 2^{-}$, and $\bar{D}^{*} \Xi_{c}$ of $J^{P}=3 / 2^{-}$. Their relevant interpolating currents are:

$$
\begin{aligned}
\eta_{1} & =\left[\delta^{a b} \bar{c}_{a} \gamma_{5} u_{b}\right]\left[\epsilon^{c d e} d_{c}^{T} \mathbb{C} \gamma_{5} s_{d} c_{e}\right] \\
& =\bar{D}^{0} \Xi_{c}^{0}, \\
\eta_{2} & =\left[\delta^{a b} \bar{c}_{a} \gamma_{v} u_{b}\right] \gamma^{v} \gamma_{5}\left[\epsilon^{c d e} d_{c}^{T} \mathbb{C} \gamma_{5} s_{d} c_{e}\right] \\
& =\bar{D}_{v}^{* 0} \gamma^{v} \gamma_{5} \Xi_{c}^{0}, \\
\eta_{3}^{\alpha} & =P_{3 / 2}^{\alpha \nu}\left[\delta^{a b} \bar{c}_{a} \gamma_{v} u_{b}\right]\left[\epsilon^{c d e} d_{c}^{T} \mathbb{C} \gamma_{5} s_{d} c_{e}\right] \\
& =P_{3 / 2}^{\alpha \nu} \bar{D}_{v}^{* 0} \Xi_{c}^{0} .
\end{aligned}
$$

In the above expressions, we have used $\mathcal{D}$ and $\mathcal{B}$ to denote the charmed meson operators $J_{\mathcal{D}}$ and the charmed baryon field $J_{\mathcal{B}} ; P_{3 / 2}^{\mu \nu}$ is the spin-3/2 projection operator

$P_{3 / 2}^{\mu \nu}=g^{\mu \nu}-\frac{1}{4} \gamma^{\mu} \gamma^{\nu}$ 
QCD sum rule studies

We use the method of QCD sum rules [40,41] to study $\bar{D}^{(*)} \Xi_{c}$ molecular states through the currents $\eta_{1,2}$ of $J^{P}=$ $1 / 2^{-}$and $\eta_{3}^{\alpha}$ of $J^{P}=3 / 2^{-}$. Taking the current $\eta_{2}$ as an example, we assume it couples to the $\bar{D}^{*} \Xi_{c}$ molecular state of $J^{P}=1 / 2^{-}$through

$\left\langle 0\left|\eta_{2}\right| X ; 1 / 2^{-}\right\rangle=f_{X} u(p)$,

where $u(p)$ is the Dirac spinor of this state, denoted as $X$ for simplicity. The two-point correlation function extracted from $\eta_{2}$ can be written as:

$$
\begin{aligned}
\Pi\left(q^{2}\right) & =i \int d^{4} x e^{i q \cdot x}\left\langle 0\left|T\left[\eta_{2}(x) \bar{\eta}_{2}(0)\right]\right| 0\right\rangle \\
& =\left(q+M_{X}\right) \Pi_{0}\left(q^{2}\right) .
\end{aligned}
$$

In QCD sum rule studies we calculate the two-point correlation function $\Pi_{0}\left(q^{2}\right)$ at both hadron and quark-gluon levels. At the hadron level, we use the dispersion relation to write it as

$\Pi_{0}\left(q^{2}\right)=\frac{1}{\pi} \int_{s_{<}}^{\infty} \frac{\operatorname{Im} \Pi_{0}(s)}{s-q^{2}-i \varepsilon} d s$,

where $s_{<}$is the physical threshold. We further define the imaginary part of the correlation function as the spectral density $\rho(s)$, which is usually evaluated at the hadron level by inserting intermediate hadron states $\sum_{n}|n\rangle\langle n|$ :

$$
\begin{aligned}
\rho_{\text {phen }}(s) & \equiv \operatorname{Im} \Pi_{0}(s) / \pi \\
& =\sum_{n} \delta\left(s-M_{n}^{2}\right)\langle 0|\eta| n\rangle\left\langle n\left|\eta^{\dagger}\right| 0\right\rangle \\
& =f_{X}^{2} \delta\left(s-m_{X}^{2}\right)+\text { continuum. }
\end{aligned}
$$

In the last step we have adopted the usual parametrization of one-pole dominance for the ground state $X$ and a continuum contribution.

At the quark-gluon level we calculate $\Pi_{0}\left(q^{2}\right)$ using the method of operator product expansion (OPE), and derive its corresponding spectral density $\rho_{\mathrm{OPE}}(s)$. After performing the Borel transformation to Eq. (11) at both hadron and quarkgluon levels, we can approximate the continuum using the spectral density above a threshold value $s_{0}$ (quark-hadron duality), and obtain the sum rule equation

$$
\Pi_{0}\left(s_{0}, M_{B}^{2}\right) \equiv f_{X}^{2} e^{-M_{X}^{2} / M_{B}^{2}}=\int_{s_{<}}^{s_{0}} e^{-s / M_{B}^{2}} \rho_{\mathrm{OPE}}(s) d s .
$$

It can be used to further calculate $M_{X}$ through

$M_{X}^{2}\left(s_{0}, M_{B}\right)=\frac{\int_{s_{<}}^{s_{0}} e^{-s / M_{B}^{2}} s \rho_{\mathrm{OPE}}(s) d s}{\int_{s_{<}}^{s_{0}} e^{-s / M_{B}^{2}} \rho_{\mathrm{OPE}}(s) d s}$.

In the present study we calculate OPEs at the leading order of $\alpha_{s}$ and up to the $D$ (imension) $=10$ terms, including the perturbative term, the charm quark mass, the quark condensates $\langle\bar{q} q\rangle /\langle\bar{s} s\rangle$, the gluon condensate $\left\langle g_{s}^{2} G G\right\rangle$, the quark-gluon mixed condensates $\left\langle g_{s} \bar{q} \sigma G q\right\rangle /\left\langle g_{s} \bar{s} \sigma G s\right\rangle$, and their combinations. We summarized the obtained spectral densities $\rho_{1 \ldots 3}(s)$ in "Appendix A", which are extracted from the currents $\eta_{1 \ldots 3}$, respectively. In the calculations we ignore the chirally suppressed terms with the up and down quark masses, and adopt the factorization assumption of vacuum saturation for higher dimensional condensates. We find that the $D=3$ quark condensates $\langle\bar{q} q\rangle /\langle\bar{s} s\rangle$ and the $D=5$ mixed condensates $\left\langle g_{s} \bar{q} \sigma G q\right\rangle /\left\langle g_{s} \bar{s} \sigma G s\right\rangle$ are multiplied by the charm quark mass, which are thus important power corrections.

\section{Numerical analyses}

We still use the current $\eta_{2}$ as an example to perform numerical analyses, where we use the following values for various QCD sum rule parameters [4,42-50]:

$$
\begin{aligned}
m_{s} & =96_{-4}^{+8} \mathrm{MeV}, \\
m_{c} & =1.275_{-0.035}^{+0.025} \mathrm{GeV}, \\
\langle\bar{q} q\rangle & =-(0.240 \pm 0.010)^{3} \mathrm{GeV}^{3}, \\
\langle\bar{s} s\rangle & =(0.8 \pm 0.1) \times\langle\bar{q} q\rangle, \\
\left\langle g_{s}^{2} G G\right\rangle & =(0.48 \pm 0.14) \mathrm{GeV}^{4}, \\
\left\langle g_{s} \bar{q} \sigma G q\right\rangle & =-M_{0}^{2} \times\langle\bar{q} q\rangle, \\
\left\langle g_{s} \bar{s} \sigma G s\right\rangle & =-M_{0}^{2} \times\langle\bar{s} s\rangle, \\
M_{0}^{2} & =(0.8 \pm 0.2) \mathrm{GeV}^{2} .
\end{aligned}
$$

Here the running mass in the $\overline{M S}$ scheme is used for the charm quark.

There are two free parameters in Eqs. (14): the Borel mass $M_{B}$ and the threshold value $s_{0}$. We use two criteria to constrain the Borel mass $M_{B}$ for a fixed $s_{0}$. The first criterion is used to insure the convergence of the OPE series. It is done by requiring the $D=10$ terms $\left(m_{c}\langle\bar{q} q\rangle^{3}\right.$ and $\left.\left\langle g_{s} \bar{q} \sigma G q\right\rangle^{2}\right)$ to be less than $20 \%$, which can determine the lower limit $M_{B}^{\text {min }}$ :

Convergence $(\mathrm{CVG}) \equiv\left|\frac{\Pi^{D=10}\left(\infty, M_{B}\right)}{\Pi\left(\infty, M_{B}\right)}\right| \leq 20 \%$.

This criterion leads to $\left(M_{B}^{\min }\right)^{2}=2.93 \mathrm{GeV}^{2}$, when setting $s_{0}=25.8 \mathrm{GeV}^{2}$.

The second criterion is used to insure the validity of onepole parametrization. It is done by requiring the pole contribution to be larger than $50 \%$, which can determine the upper limit $M_{B}^{\max }$ :

Pole Contribution $(\mathrm{PC}) \equiv \frac{\Pi\left(s_{0}, M_{B}\right)}{\Pi\left(\infty, M_{B}\right)} \geq 50 \%$

This criterion leads to $\left(M_{B}^{\max }\right)^{2}=3.07 \mathrm{GeV}^{2}$, when setting $s_{0}=25.8 \mathrm{GeV}^{2}$. 


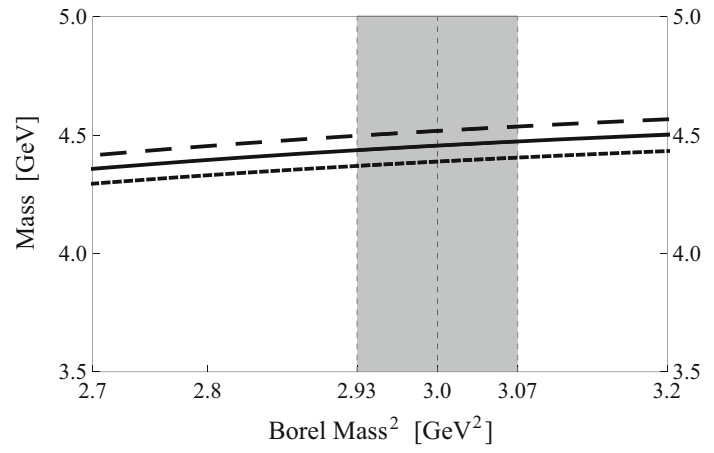

Fig. 2 The variation of $M_{X}$ with respect to the Borel mass $M_{B}$, calculated using the current $\eta_{2}$. The short-dashed, solid, and long-dashed curves are obtained by setting $s_{0}=24.8,25.8$, and $26.8 \mathrm{GeV}^{2}$, respectively

Altogether we extract the working region of Borel mass to be $2.93 \mathrm{GeV}^{2}<M_{B}^{2}<3.07 \mathrm{GeV}^{2}$ for the current $\eta_{2}$ with the threshold value $s_{0}=25.8 \mathrm{GeV}^{2}$. We show the variation of $M_{X}$ with respect to the Borel mass $M_{B}$ in Fig. 2 in a broader region $2.7 \mathrm{GeV}^{2} \leq M_{B}^{2} \leq 3.2 \mathrm{GeV}^{2}$, and find it more stable inside the above Borel window.

Redoing the same procedures by changing $s_{0}$, we find that there are non-vanishing Borel windows as long as $s_{0} \geq$ $s_{0}^{\min }=24.8 \mathrm{GeV}^{2}$. Accordingly, we choose the threshold value $s_{0}$ to be about $1.0 \mathrm{GeV}$ larger with the uncertainty $\pm 1.0 \mathrm{GeV}$, i.e., $24.8 \mathrm{GeV}^{2} \leq s_{0} \leq 26.8 \mathrm{GeV}^{2}$. Altogether, our working regions for the current $\eta_{2}$ are determined to be $24.8 \mathrm{GeV}^{2} \leq s_{0} \leq 26.8 \mathrm{GeV}^{2}$ and $2.93 \mathrm{GeV}^{2}<M_{B}^{2}<$ $3.07 \mathrm{GeV}^{2}$, where the mass is extracted to be:

$M_{\bar{D}^{*} \Xi_{c} ; 1 / 2^{-}}=4.46_{-0.14}^{+0.16} \mathrm{GeV}$.

Here the central value corresponds to $M_{B}^{2}=3.00 \mathrm{GeV}^{2}$ and $s_{0}=25.8 \mathrm{GeV}^{2}$. Its uncertainty comes from the Borel mass $M_{B}$, the threshold value $s_{0}$, the charm quark mass $m_{c}$, and various QCD sum rule parameters listed in Eqs. (15). This mass value is consistent with the experimental mass of the $P_{c s}(4459)^{0}$, supporting its interpretation as the $\bar{D}^{*} \Xi_{c}$ molecular state of $J^{P}=1 / 2^{-}$.

Similarly, we use the currents $\eta_{1}$ and $\eta_{3}^{\alpha}$ to perform numerical analyses, and extract masses of the $J^{P}=1 / 2^{-} \bar{D} \Xi_{c}$ molecular state and the $J^{P}=3 / 2^{-} \bar{D}^{*} \Xi_{c}$ molecular state to be:

$$
\begin{aligned}
M_{\bar{D} \Xi_{c} ; 1 / 2^{-}} & =4.29_{-0.12}^{+0.13} \mathrm{GeV}, \\
M_{\bar{D}^{*} \Xi_{c} ; 3 / 2^{-}} & =4.47_{-0.15}^{+0.19} \mathrm{GeV} .
\end{aligned}
$$

Hence, our results also support the interpretation of the $P_{C S}(4459)^{0}$ as the $\bar{D}^{*} \Xi_{c}$ molecular state of $J^{P}=3 / 2^{-}$. We summarize all the above results in Table 1 .

Generally speaking, understanding the nature of exotic hadrons is a complicated topic, since different structures with the same quantum numbers can contribute to the same state, and different structures may have similar masses. However, these different structures may lead to different decay processes. Therefore, to determine whether the $P_{c s}(4459)^{0}$ is the $\bar{D}^{*} \Xi_{c}$ molecular state of $J^{P}=1 / 2^{-}$or the one of $J^{P}=3 / 2^{-}$in our framework, we shall further calculate its width in our future study, to be compared with its experimental value $\Gamma_{P_{c s}(4459)^{0}}=17.3 \pm 6.5_{-5.7}^{+8.0} \mathrm{MeV}$ [3].

\section{Summary and discussions}

Very recently, the LHCb Collaboration reported the evidence of a hidden-charm pentaquark state with strangeness, $P_{c s}(4459)^{0}$, in the $J / \psi \Lambda$ invariant mass spectrum of the $\Xi_{b}^{-} \rightarrow J / \psi K^{-} \Lambda$ decays [3]. This state contains at least five quarks $\bar{c} \operatorname{cs} q q(q=u / d)$, with one of them the strange quark. This LHCb experiment indicates that there probably exist many more exotic hadrons with strangeness to be discovered in the near future, so a new hadron spectrum is waiting to be constructed.

The $P_{c s}(4459)^{0}$ is about $19 \mathrm{MeV}$ below the $\bar{D}^{* 0} \Xi_{c}^{0}$ threshold, so it is natural to interpret it as the $\bar{D}^{*} \Xi_{c}$ molecular state. Accordingly, in this letter we use the method of QCD sum rules to study the $\bar{D}^{*} \Xi_{c}$ molecular states of $J^{P}=1 / 2^{-}$and $3 / 2^{-}$, and at the same time we also study the $\bar{D} \Xi_{c}$ molecular state of $J^{P}=1 / 2^{-}$. We evaluate their masses to be

$$
\begin{aligned}
M_{\bar{D} \Xi_{c} ; 1 / 2^{-}} & =4.29_{-0.12}^{+0.13} \mathrm{GeV}, \\
M_{\bar{D}^{*} \Xi_{c} ; 1 / 2^{-}} & =4.46_{-0.14}^{+0.16} \mathrm{GeV}, \\
M_{\bar{D}^{*} \Xi_{c} ; 3 / 2^{-}} & =4.47_{-0.15}^{+0.19} \mathrm{GeV} .
\end{aligned}
$$

Hence, our QCD sum rule results support the interpretation of the $P_{c S}(4459)^{0}$ as the $\bar{D}^{*} \Xi_{c}$ molecular state of either $J^{P}=$ $1 / 2^{-}$or $3 / 2^{-}$.

Within the hadronic molecular picture, the three $\mathrm{LHCb}$ experiments observing $P_{c}$ and $P_{c s}$ states [1-3] can be well understood as a whole:

- The transition of $\Xi_{b}^{-} \rightarrow J / \psi K^{-} \Lambda$ is dominated by the Cabibbo-favored weak decay of $b \rightarrow c+\bar{c} s$ via the $V-A$ current. This leads to an intuitive expectation that the $s$ and $d$ pair in $\Xi_{b}^{-}$may exist as a spectator, so that their total spin $S=0$ is conserved. As a consequence, if this $s d$ pair is to be combined with a $c$ quark to form a charmed baryon, it will favor the $\Xi_{c}$ instead of the $\Xi_{c}^{\prime}$ and $\Xi_{c}^{*}$, of which the total spin of the $s d$ pair is $S=1$. Accordingly, the $P_{c S}(4459)^{0}$ possibly as the $\bar{D}^{*} \Xi_{c}$ molecular state was observed in this channel by LHCb [3], other than those possibly existing $\bar{D}^{(*)} \Xi_{c}^{\prime}$ and $\bar{D}^{(*)} \Xi_{c}^{*}$ molecular states. We illustrate this process in Fig. 3(a).

- The transition of $\Lambda_{b}^{0} \rightarrow J / \psi K^{-} p$ can be similarly analysed, which may favor the $\Lambda_{c}$ instead of the $\Sigma_{c}$ and $\Sigma_{c}^{*}$. However, in this case the $\Lambda_{c}$ is probably not bounded with 
Table 1 Masses of the $\bar{D}^{(*)} \Xi_{c}$ hadronic molecular states, extracted from the currents $\eta_{1 \ldots 3}^{(\alpha)}$

\begin{tabular}{|c|c|c|c|c|c|c|}
\hline \multirow[t]{2}{*}{ Currents } & \multirow[t]{2}{*}{ Configuration } & \multirow[t]{2}{*}{$s_{0}^{\min }\left[\mathrm{GeV}^{2}\right]$} & \multicolumn{2}{|c|}{ Working Regions } & \multirow[t]{2}{*}{ Pole [\%] } & \multirow[t]{2}{*}{ Mass $[\mathrm{GeV}$} \\
\hline & & & $s_{0}\left[\mathrm{GeV}^{2}\right]$ & $M_{B}^{2}\left[\mathrm{GeV}^{2}\right]$ & & \\
\hline$\eta_{1}$ & $\left|\bar{D} \Xi_{c} ; 1 / 2^{-}\right\rangle$ & 22.3 & $23.3 \pm 1.0$ & $2.61-2.77$ & $50-56$ & $4.29_{-0.12}^{+0.13}$ \\
\hline$\eta_{2}$ & $\left|\bar{D}^{*} \Xi_{c} ; 1 / 2^{-}\right\rangle$ & 24.8 & $25.8 \pm 1.0$ & $2.93-3.07$ & $50-55$ & $4.46_{-0.14}^{+0.16}$ \\
\hline$\eta_{3}^{\alpha}$ & $\left|\bar{D}^{*} \Xi_{c} ; 3 / 2^{-}\right\rangle$ & 24.4 & $25.4 \pm 1.0$ & $2.81-2.95$ & $50-55$ & $4.47_{-0.15}^{+0.19}$ \\
\hline
\end{tabular}

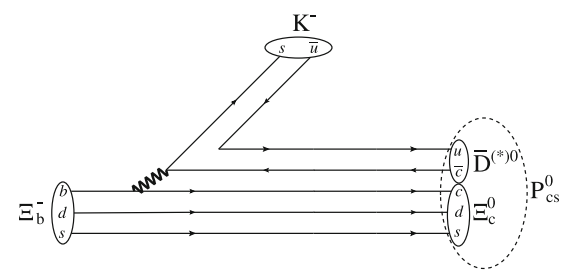

(a) $\Xi_{b}^{-} \rightarrow \bar{D}^{(*) 0} \Xi_{c}^{0} K^{-}$

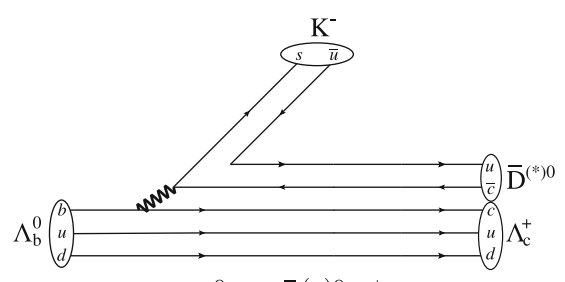

(b) $\Lambda_{b}^{0} \rightarrow \bar{D}^{(*) 0} \Lambda_{c}^{+} K^{-}$

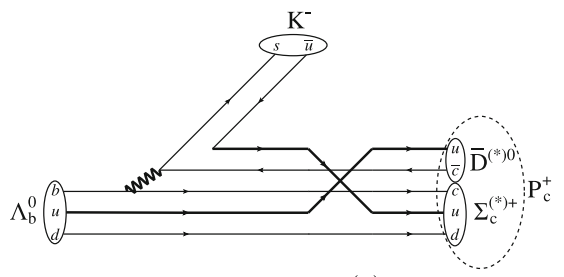

(c) $\Lambda_{b}^{0} \rightarrow \bar{D}^{(*) 0} \Sigma_{c}^{(*)+} K^{-}$

Fig. 3 Possible production mechanisms of the $P_{c}^{+}$and $P_{c s}^{0}$ states in $\Lambda_{b}^{0} / \Xi_{b}^{-}$decays

the charmed mesons due to the lack of $\pi$ exchanges. It is only because of the significantly larger data sample (680k for the $\Lambda_{b}^{0} \rightarrow J / \psi K^{-} p$ decays and only $4 \mathrm{k}$ for the $\Xi_{b}^{-} \rightarrow J / \psi K^{-} \Lambda$ decays [3]), that the $P_{c}(4312)$, $P_{c}(4440)$, and $P_{c}(4457)$ possibly as the $\bar{D} \Sigma_{c}$ and $\bar{D}^{*} \Sigma_{c}$ molecular states were observed in this channel by $\mathrm{LHCb}$ $[1,2]$. We illustrate the relevant two processes in Fig. 3b, c.

Therefore, the three LHCb experiments observing $P_{c}$ and $P_{c s}$ states [1-3] strongly support the hadronic molecular picture and the existence of "hadronic molecules".

To further verify the above hadronic molecular picture, we propose to investigate the $P_{c s}(4459)^{0}$ in future experiments to examine whether it can be separated into two states. We also propose to search for the $\bar{D} \Xi_{c}$ molecular state of $J^{P}=1 / 2^{-}$, whose mass is predicted to be $4.29_{-0.12}^{+0.13} \mathrm{GeV}$. If the hadronic molecular picture turns out to be correct, our understanding on the construction of the subatomic world be significantly improved, and our understanding on the nonperturbative behaviors of the strong interaction at the low energy region would also be significantly improved.

Acknowledgements This project is supported by the National Natural Science Foundation of China under Grants No. 11722540, No. 11975165, and No. 12075019, the China National Funds for Distinguished Young Scientists under Grant No. 11825503, the National Program for Support of Top-notch Young Professionals, and the Fundamental Research Funds for the Central Universities.

Data Availability Statement This manuscript has no associated data or the data will not be deposited. [Authors' comment: All data generated during this study are contained in this published article.]
Open Access This article is licensed under a Creative Commons Attribution 4.0 International License, which permits use, sharing, adaptation, distribution and reproduction in any medium or format, as long as you give appropriate credit to the original author(s) and the source, provide a link to the Creative Commons licence, and indicate if changes were made. The images or other third party material in this article are included in the article's Creative Commons licence, unless indicated otherwise in a credit line to the material. If material is not included in the article's Creative Commons licence and your intended use is not permitted by statutory regulation or exceeds the permitted use, you will need to obtain permission directly from the copyright holder. To view a copy of this licence, visit http://creativecomm ons.org/licenses/by/4.0/.

Funded by $\mathrm{SCOAP}^{3}$.

\section{Appendix: Spectral densities}

In this appendix we list the spectral densities $\rho_{1 \ldots 3}(s)$ extracted for the currents $\eta_{1 \ldots 3}$. In the following expressions, $\mathcal{F}(s)=\left[(\alpha+\beta) m_{c}^{2}-\alpha \beta s\right], \mathcal{H}(s)=\left[m_{c}^{2}-\alpha(1-\alpha) s\right]$, and the integration limits are $\alpha_{\min }=\frac{1-\sqrt{1-4 m_{c}^{2} / s}}{2}, \alpha_{\max }=$ $\frac{1+\sqrt{1-4 m_{c}^{2} / s}}{2}, \beta_{\min }=\frac{\alpha m_{c}^{2}}{\alpha s-m_{c}^{2}}$, and $\beta_{\max }=1-\alpha$. In the calculations we take into account both the $m_{s}$ and $m_{s}^{2}$ terms, but here we list only the $m_{s}$ terms.

The spectral density $\rho_{1}(s)$ extracted for the current $\eta_{1}$ is

$$
\begin{aligned}
\rho_{1}(s)= & \rho_{1}^{\text {pert }}(s)+\rho_{1}^{\langle\bar{q} q\rangle}(s)+\rho_{1}^{\langle G G\rangle}(s)+\rho_{1}^{\langle\bar{q} G q\rangle}(s)+\rho_{1}^{\langle\bar{q} q\rangle^{2}}(s) \\
& +\rho_{1}^{\langle\bar{q} q\rangle\langle\bar{q} G q\rangle}(s)+\rho_{1}^{\langle\bar{q} G q\rangle^{2}}(s)+\rho_{1}^{\langle\bar{q} q\rangle^{3}}(s)
\end{aligned}
$$

where 
409 Page 6 of 9

Eur. Phys. J. C (2021) 81:409

$$
\begin{aligned}
& \rho_{1}^{\text {pert }}(s)=\int_{\alpha_{\min }}^{\alpha_{\max }} d \alpha \int_{\beta_{\min }}^{\beta_{\max }} d \beta\left\{\mathcal{F}(s)^{5} \times \frac{(1-\alpha-\beta)^{3}}{163840 \pi^{8} \alpha^{5} \beta^{4}}\right\}, \\
& \rho_{1}^{\langle\bar{q} q\rangle}(s)=\int_{\alpha_{\min }}^{\alpha_{\max }} d \alpha \int_{\beta_{\min }}^{\beta_{\max }} d \beta\left\{\mathcal{F}(s)^{3}\right. \\
& \left.\times \frac{(1-\alpha-\beta)\left((\alpha+\beta-1) \beta m_{c}\langle\bar{q} q\rangle+\beta m_{s}(\langle\bar{s} s\rangle-2\langle\bar{q} q\rangle)\right)}{2048 \pi^{6} \alpha^{3} \beta^{3}}\right\}, \\
& \rho_{1}^{\langle G G\rangle}(s)=\left\langle g_{s}^{2} G G\right\rangle \int_{\alpha_{\min }}^{\alpha_{\max }} d \alpha \int_{\beta_{\min }}^{\beta_{\max }} d \beta\left\{m_{c}^{2} \mathcal{F}(s)^{2}\right. \\
& \times \frac{(1-\alpha-\beta)^{3}\left(\alpha^{3}+\beta^{3}\right)}{196608 \pi^{8} \alpha^{5} \beta^{4}} \\
& \left.+\mathcal{F}(s)^{3} \times \frac{(\alpha+\beta-1)\left(3 \alpha^{3}-\alpha^{2}(\beta+3)-2 \alpha(\beta-1) \beta-(\beta-1)^{2} \beta\right)}{196608 \pi^{8} \alpha^{5} \beta^{3}}\right\}, \\
& \rho_{1}^{\langle\bar{q} G q\rangle}(s)=\int_{\alpha_{\min }}^{\alpha_{\max }} d \alpha \int_{\beta_{\min }}^{\beta_{\max }} d \beta\left\{\mathcal{F}(s)^{2}\right. \\
& \left.\times \frac{-3\left(\alpha^{2}+\alpha(3 \beta-2)+2 \beta^{2}-3 \beta+1\right) m_{c}\left\langle g_{s} \bar{q} \sigma G q\right\rangle+3 \beta^{2} m_{s}\left\langle g_{s} \bar{q} \sigma G q\right\rangle}{4096 \pi^{6} \alpha^{2} \beta^{3}}\right\}, \\
& \rho_{1}^{\langle\bar{q} q\rangle^{2}}(s)=\int_{\alpha_{\min }}^{\alpha_{\max }} d \alpha \int_{\beta_{\min }}^{\beta_{\max }} d \beta\left\{\frac{-\mathcal{F}(s)^{2} \times\langle\bar{q} q\rangle\langle\bar{s} s\rangle+m_{c} m_{s} \mathcal{F}(s) \times \alpha\left(2\langle\bar{q} q\rangle^{2}-\langle\bar{q} q\rangle\langle\bar{s} s\rangle\right)}{256 \pi^{4} \alpha^{2} \beta}\right\}, \\
& \rho_{1}^{\langle\bar{q} q\rangle\langle\bar{q} G q\rangle}(s)=\int_{\alpha_{\min }}^{\alpha_{\max }} d \alpha\left\{\int_{\beta_{\min }}^{\beta_{\max }} d \beta\left\{\frac{m_{c} m_{s}\left\langle g_{s} \bar{q} \sigma G q\right\rangle(2\langle\bar{q} q\rangle-\langle\bar{s} s\rangle)}{512 \pi^{4} \beta}\right\}\right. \\
& +\mathcal{H}(s) \times \frac{\langle\bar{q} q\rangle\left\langle g_{s} \bar{s} \sigma G s\right\rangle+\langle\bar{s} s\rangle\left\langle g_{s} \bar{q} \sigma G q\right\rangle}{512 \pi^{4} \alpha} \\
& \left.-\frac{m_{c} m_{s}\left\langle g_{s} \bar{q} \sigma G q\right\rangle(4\langle\bar{q} q\rangle-\langle\bar{s} s\rangle)}{1024 \pi^{4}}\right\} \\
& \rho_{1}^{\langle\bar{q} G q\rangle^{2}}(s)=\int_{\alpha_{\min }}^{\alpha_{\max }} d \alpha\left\{\frac{(\alpha-1)\left\langle g_{s} \bar{q} \sigma G q\right\rangle\left\langle g_{s} \bar{s} \sigma G s\right\rangle}{1024 \pi^{4}}\right\} \\
& +\int_{0}^{1} d \alpha\left\{\delta\left(s-\frac{m_{c}^{2}}{\alpha(1-\alpha)}\right)\right. \\
& \left.\times \frac{\left\langle g_{s} \bar{q} \sigma G q\right\rangle\left(m_{c}^{3} m_{s}\left\langle g_{s} \bar{q} \sigma G q\right\rangle / M_{B}^{2}+(1-\alpha) m_{c}^{2}\left\langle g_{s} \bar{s} \sigma G s\right\rangle-\alpha(\alpha+1) m_{c} m_{s}\left\langle g_{s} \bar{q} \sigma G q\right\rangle\right)}{2048 \pi^{4}(\alpha-1) \alpha}\right\}, \\
& \rho_{1}^{\langle\bar{q} q\rangle^{3}}(s)=m_{c}\langle\bar{q} q\rangle^{2}\langle\bar{s} s\rangle \int_{\alpha_{\min }}^{\alpha_{\max }} d \alpha\left\{\frac{1}{96 \pi^{2}}\right\} .
\end{aligned}
$$

The spectral density $\rho_{2}(s)$ extracted for the current $\eta_{2}$ is

$$
\begin{aligned}
\rho_{2}(s)= & \rho_{2}^{\text {pert }}(s)+\rho_{2}^{\langle\bar{q} q\rangle}(s)+\rho_{2}^{\langle G G\rangle}(s)+\rho_{2}^{\langle\bar{q} G q\rangle}(s)+\rho_{2}^{\langle\bar{q} q\rangle^{2}}(s) \\
& +\rho_{2}^{\langle\bar{q} q\rangle\langle\bar{q} G q\rangle}(s)+\rho_{2}^{\langle\bar{q} G q\rangle^{2}}(s)+\rho_{2}^{\langle\bar{q} q\rangle^{3}}(s),
\end{aligned}
$$

Springer 
Eur. Phys. J. C (2021) 81:409

Page 7 of 9

409

where

$$
\begin{aligned}
\rho_{2}^{\text {pert }}(s)= & \int_{\alpha_{\min }}^{\alpha_{\max }} d \alpha \int_{\beta_{\min }}^{\beta_{\max }} d \beta\left\{\mathcal{F}(s)^{5} \times \frac{(1-\alpha-\beta)^{3}}{\left.81920 \pi^{8} \alpha^{5} \beta^{4}\right\}}\right. \\
\rho_{2}^{\langle\bar{q} q\rangle}(s)= & \int_{\alpha_{\min }}^{\alpha_{\max }} d \alpha \int_{\beta_{\min }}^{\beta_{\max }} d \beta\left\{\mathcal{F}(s)^{3}\right. \\
& \left.\times \frac{(1-\alpha-\beta)\left(2(\alpha+\beta-1) m_{c}\langle\bar{q} q\rangle+\beta m_{s}(\langle\bar{s} s\rangle-2\langle\bar{q} q\rangle)\right)}{1024 \pi^{6} \alpha^{3} \beta^{3}}\right\}, \\
\rho_{2}^{\langle G G\rangle}(s)= & \left\langle g_{s}^{2} G G\right\rangle \int_{\alpha_{\min }}^{\alpha_{\max }} d \alpha \int_{\beta_{\min }}^{\beta_{\max }} d \beta\left\{m_{c}^{2} \mathcal{F}(s)^{2} \times \frac{(1-\alpha-\beta)^{3}\left(\alpha^{3}+\beta^{3}\right)}{98304 \pi^{8} \alpha^{5} \beta^{4}}\right. \\
& \left.+\mathcal{F}(s)^{3} \times \frac{(1-\alpha-\beta)\left(3 \alpha^{3}+\alpha^{2}(7 \beta-3)+2 \alpha(\beta-1) \beta+(\beta-1)^{2} \beta\right)}{98304 \pi^{8} \alpha^{5} \beta^{3}}\right\}, \\
\rho_{2}^{\langle\bar{q} G q\rangle}(s)= & \int_{\alpha_{\min }}^{\alpha_{\max }} d \alpha \int_{\beta_{\min }}^{\beta_{\max }} d \beta\left\{\mathcal{F}(s)^{2} \times \frac{-3\left\langle g_{s} \bar{q} \sigma G q\right\rangle\left(2(\alpha+\beta-1) m_{c}-\beta m_{s}\right)}{2048 \pi^{6} \alpha^{2} \beta^{2}}\right\}, \\
\rho_{2}^{\langle\bar{q} q\rangle^{2}}(s)= & \int_{\alpha_{\min }}^{\alpha_{\max }} d \alpha \int_{\beta_{\min }}^{\beta_{\max }} d \beta\left\{\frac{-\mathcal{F}(s)^{2} \times\langle\bar{q} q\rangle\langle\bar{s} s\rangle-m_{c} m_{s} \mathcal{F}(s) \times 2 \alpha(\langle\bar{s} s\rangle-2\langle\bar{q} q\rangle)}{128 \pi^{4} \alpha^{2} \beta}\right\}, \\
\rho_{2}^{\langle\bar{q} q\rangle\langle\bar{q} G q\rangle}(s)= & \int_{\alpha_{\min }}^{\alpha_{\max }} d \alpha\{\mathcal{H}(s) \\
& \left.\times \frac{\langle\bar{q} q\rangle\left\langle g_{s} \bar{s} \sigma G s\right\rangle+\langle\bar{s} s\rangle\left\langle g_{s} \bar{q} \sigma G q\right\rangle}{256 \pi^{4} \alpha}-\frac{m_{c} m_{s}\left\langle g_{s} \bar{q} \sigma G q\right\rangle(4\langle\bar{q} q\rangle-\langle\bar{s} s\rangle)}{256 \pi^{4}}\right\}, \\
\rho_{2}^{\langle\bar{q} G q\rangle^{2}}(s)= & \int_{\alpha_{\min }}^{\alpha_{\max }} d \alpha\left\{\frac{(\alpha-1)\left\langle g_{s} \bar{q} \sigma G q\right\rangle\left\langle g_{s} \bar{s} \sigma G s\right\rangle}{512 \pi^{4}}\right\}+\int_{0}^{1} d \alpha\left\{\delta\left(s-\frac{m_{c}^{2}}{\alpha(1-\alpha)}\right)\right. \\
& \left.\times \frac{\left\langle g_{s} \bar{q} \sigma G q\right\rangle\left(2 m_{c}^{3} m_{s}\left\langle g_{s} \bar{q} \sigma G q\right\rangle / M_{B}^{2}+(1-\alpha) m_{c}^{2}\left\langle g_{s} \bar{s} \sigma G s\right\rangle-2(\alpha-1) \alpha m_{s}\left\langle g_{s} \bar{q} \sigma G q\right\rangle\right)}{1024 \pi^{4}(\alpha-1) \alpha}\right\}, \\
\rho_{2}^{\langle\bar{q} q\rangle^{3}}(s)= & m_{c}\langle\bar{q} q\rangle^{2}\langle\bar{s} s\rangle \int_{\alpha_{\min }}^{\alpha_{\max }} d \alpha\left\{\frac{1}{\left.24 \pi^{2}\right\} .}\right.
\end{aligned}
$$

The spectral density $\rho_{3}(s)$ extracted for the current $\eta_{3}^{\alpha}$ is

$$
\begin{aligned}
\rho_{3}(s)= & \rho_{3}^{\text {pert }}(s)+\rho_{3}^{\langle\bar{q} q\rangle}(s)+\rho_{3}^{\langle G G\rangle}(s)+\rho_{3}^{\langle\bar{q} G q\rangle}(s)+\rho_{3}^{\langle\bar{q} q\rangle^{2}}(s) \\
& +\rho_{3}^{\langle\bar{q} q\rangle\langle\bar{q} G q\rangle}(s)+\rho_{3}^{\langle\bar{q} G q\rangle^{2}}(s)+\rho_{3}^{\langle\bar{q} q\rangle^{3}}(s),
\end{aligned}
$$

Springer 
409 Page 8 of 9

Eur. Phys. J. C (2021) 81:409

where

$$
\begin{aligned}
& \rho_{3}^{\text {pert }}(s)=\int_{\alpha_{\min }}^{\alpha_{\max }} d \alpha \int_{\beta_{\min }}^{\beta_{\max }} d \beta\left\{\mathcal{F}(s)^{5}\right. \\
& \left.\times \frac{(1-\alpha-\beta)^{3}(\alpha+\beta+4)}{1310720 \pi^{8} \alpha^{5} \beta^{4}}\right\}, \\
& \rho_{3}^{\langle\bar{q} q\rangle}(s)=\int_{\alpha_{\min }}^{\alpha_{\max }} d \alpha \int_{\beta_{\min }}^{\beta_{\max }} d \beta\left\{\mathcal{F}(s)^{3}\right. \\
& \left.\times \frac{(\alpha+\beta-1)\left(6(1-\alpha-\beta) m_{c}\langle\bar{q} q\rangle+\beta(2 \alpha+2 \beta+3) m_{s}(2\langle\bar{q} q\rangle-\langle\bar{s} s\rangle)\right)}{16384 \pi^{6} \alpha^{3} \beta^{3}}\right\}, \\
& \rho_{3}^{\langle G G\rangle}(s)=\left\langle g_{s}^{2} G G\right\rangle \int_{\alpha_{\min }}^{\alpha_{\max }} d \alpha \int_{\beta_{\min }}^{\beta_{\max }} d \beta\left\{m_{c}^{2} \mathcal{F}(s)^{2}\right. \\
& \times \frac{(1-\alpha-\beta)^{3}(\alpha+\beta+4)\left(\alpha^{3}+\beta^{3}\right)}{1572864 \pi^{8} \alpha^{5} \beta^{4}} \\
& +\mathcal{F}(s)^{3} \times\left(\frac{4 \alpha^{5}-3 \alpha^{4}(3 \beta+11)-6 \alpha^{3}\left(6 \beta^{2}+13 \beta-9\right)-\alpha^{2}\left(32 \beta^{3}+51 \beta^{2}-108 \beta+25\right)}{4718592 \pi^{8} \alpha^{5} \beta^{3}}\right. \\
& \left.\left.\times \frac{-3 \alpha(\beta-1)^{2} \beta(4 \beta+11)-3(\beta-1)^{3} \beta(\beta+4)}{4718592 \pi^{8} \alpha^{5} \beta^{3}}\right)\right\}, \\
& \rho_{3}^{\langle\bar{q} G q\rangle}(s)=\int_{\alpha_{\min }}^{\alpha_{\max }} d \alpha \int_{\beta_{\min }}^{\beta_{\max }} d \beta\left\{\mathcal{F}(s)^{2}\right. \\
& \left.\times \frac{3\left\langle g_{s} \bar{q} \sigma G q\right\rangle\left(-6(\alpha+\beta-1) m_{c}+\beta(4 \alpha+4 \beta+1) m_{s}\right)}{32768 \pi^{6} \alpha^{2} \beta^{2}}\right\}, \\
& \rho_{3}^{\langle\bar{q} q\rangle^{2}}(s)=\int_{\alpha_{\min }}^{\alpha_{\max }} d \alpha \int_{\beta_{\min }}^{\beta_{\max }} d \beta\left\{\frac{-\mathcal{F}(s)^{2} \times(4 \alpha+4 \beta+1)\langle\bar{q} q\rangle\langle\bar{s} s\rangle-m_{c} m_{s} \mathcal{F}(s) \times 6 \alpha(\langle\bar{s} s\rangle-2\langle\bar{q} q\rangle)}{2048 \pi^{4} \alpha^{2} \beta}\right\}, \\
& \rho_{3}^{\langle\bar{q} q\rangle\langle\bar{q} G q\rangle}(s)=\int_{\alpha_{\min }}^{\alpha_{\max }} d \alpha\left\{\int_{\beta_{\min }}^{\beta_{\max }} d \beta\left\{\mathcal{F}(s) \times \frac{-\langle\bar{q} q\rangle\left\langle g_{s} \bar{s} \sigma G s\right\rangle-\langle\bar{s} s\rangle\left\langle g_{s} \bar{q} \sigma G q\right\rangle}{1024 \pi^{4} \alpha}\right\}\right. \\
& \left.+\mathcal{H}(s) \times \frac{5\langle\bar{q} q\rangle\left\langle g_{s} \bar{s} \sigma G s\right\rangle+5\langle\bar{s} s\rangle\left\langle g_{s} \bar{q} \sigma G q\right\rangle}{4096 \pi^{4} \alpha}-\frac{3 m_{c} m_{s}\left\langle g_{s} \bar{q} \sigma G q\right\rangle(4\langle\bar{q} q\rangle-\langle\bar{s} s\rangle)}{4096 \pi^{4}}\right\}, \\
& \rho_{3}^{\langle\bar{q} G q\rangle^{2}}(s)=\int_{\alpha_{\min }}^{\alpha_{\max }} d \alpha\left\{\frac{3(\alpha-1)\left\langle g_{s} \bar{q} \sigma G q\right\rangle\left\langle g_{s} \bar{s} \sigma G s\right\rangle}{8192 \pi^{4}}\right\}+\int_{0}^{1} d \alpha\left\{\delta\left(s-\frac{m_{c}^{2}}{\alpha(1-\alpha)}\right)\right. \\
& \left.\times \frac{\left\langle g_{s} \bar{q} \sigma G q\right\rangle\left(6 m_{c}^{3} m_{s}\left\langle g_{s} \bar{q} \sigma G q\right\rangle / M_{B}^{2}-5(\alpha-1) m_{c}^{2}\left\langle g_{s} \bar{s} \sigma G s\right\rangle-6(\alpha-1) \alpha m_{c} m_{s}\left\langle g_{s} \bar{q} \sigma G q\right\rangle\right)}{16384 \pi^{4}(\alpha-1) \alpha}\right\}, \\
& \rho_{3}^{\langle\bar{q} q\rangle^{3}}(s)=m_{c}\langle\bar{q} q\rangle^{2}\langle\bar{s} s\rangle \int_{\alpha_{\min }}^{\alpha_{\max }} d \alpha\left\{\frac{1}{128 \pi^{2}}\right\}
\end{aligned}
$$

Springer 


\section{References}

1. R. Aaij et al. (LHCb Collaboration), Phys. Rev. Lett. 115, 072001 (2015)

2. R. Aaij et al. (LHCb Collaboration), Phys. Rev. Lett. 122, 222001 (2019)

3. R. Aaij et al. [LHCb Collaboration], (2012). arXiv:2012.10380 [hep-ex]

4. M. Tanabashi et al. (Particle Data Group), Phys. Rev. D 98, 030001 (2018)

5. M. Gell-Mann, Phys. Lett. 8, 214 (1964)

6. G. Zweig, CERN-TH-401 (1981)

7. R. Chen, X. Liu, X.Q. Li, S.L. Zhu, Phys. Rev. Lett. 115, 132002 (2015)

8. H.X. Chen, W. Chen, X. Liu, T.G. Steele, S.L. Zhu, Phys. Rev. Lett. 115, 172001 (2015)

9. L. Roca, J. Nieves, E. Oset, Phys. Rev. D 92, 094003 (2015)

10. U.G. Meissner, J.A. Oller, Phys. Lett. B 751, 59 (2015)

11. M.Z. Liu, Y.W. Pan, F.Z. Peng, M.S. Sánchez, L.S. Geng, A. Hosaka, M.P. Valderrama, Phys. Rev. Lett. 122, 242001 (2019)

12. J.J. Wu, R. Molina, E. Oset, B.S. Zou, Phys. Rev. Lett. 105, 232001 (2010)

13. W.L. Wang, F. Huang, Z.Y. Zhang, B.S. Zou, Phys. Rev. C 84, $015203(2011)$

14. Z.C. Yang, Z.F. Sun, J. He, X. Liu, S.L. Zhu, Chin. Phys. C 36, 6 (2012)

15. X.Q. Li, X. Liu, Eur. Phys. J. C 74, 3198 (2014)

16. M. Karliner, J.L. Rosner, Phys. Rev. Lett. 115, 122001 (2015)

17. L. Maiani, A.D. Polosa, V. Riquer, Phys. Lett. B 749, 289 (2015)

18. R.F. Lebed, Phys. Lett. B 749, 454 (2015)

19. Z.G. Wang, Eur. Phys. J. C 76, 70 (2016)

20. F.K. Guo, U.G. Meissner, W. Wang, Z. Yang, Phys. Rev. D 92, 071502(R) (2015)

21. X.H. Liu, Q. Wang, Q. Zhao, Phys. Lett. B 757, 231 (2016)

22. M. Bayar, F. Aceti, F.K. Guo, E. Oset, Phys. Rev. D 94, 074039 (2016)

23. H.X. Chen, W. Chen, X. Liu, S.L. Zhu, Phys. Rept. 639, 1 (2016)

24. Y.R. Liu, H.X. Chen, W. Chen, X. Liu, S.L. Zhu, Prog. Part. Nucl. Phys. 107, 237 (2019)

25. F.K. Guo, C. Hanhart, U.G. Meißner, Q. Wang, Q. Zhao, B.S. Zou, Rev. Mod. Phys. 90, 015004 (2018)
26. A. Ali, J.S. Lange, S. Stone, Prog. Part. Nucl. Phys. 97, 123 (2017)

27. S.L. Olsen, T. Skwarnicki, D. Zieminska, Rev. Mod. Phys. 90, 015003 (2018)

28. N. Brambilla, S. Eidelman, C. Hanhart, A. Nefediev, C.P. Shen, C.E. Thomas, A. Vairo, C.Z. Yuan, Phys. Rept. 873, 1 (2020)

29. H.Y. Cheng, C.K. Chua, Phys. Rev. D 92, 096009 (2015)

30. V.V. Anisovich, M.A. Matveev, J. Nyiri, A.V. Sarantsev, A.N. Semenova, Int. J. Mod. Phys. A 30, 1550190 (2015)

31. Z.G. Wang, Eur. Phys. J. C 76, 142 (2016)

32. A. Feijoo, V.K. Magas, A. Ramos, E. Oset, Eur. Phys. J. C 76, 446 (2016)

33. J.X. Lu, E. Wang, J.J. Xie, L.S. Geng, E. Oset, Phys. Rev. D 93, 094009 (2016)

34. R. Chen, J. He, X. Liu, Chin. Phys. C 41, 103105 (2017)

35. C.W. Xiao, J. Nieves, E. Oset, Phys. Lett. B 799, 135051 (2019)

36. Q. Zhang, B.R. He, J.L. Ping, (2020). arXiv:2006.01042 [hep-ph]

37. C.W. Shen, H.J. Jing, F.K. Guo, J.J. Wu, Symmetry 12, 1611 (2020)

38. H.X. Chen, L.S. Geng, W.H. Liang, E. Oset, E. Wang, J.J. Xie, Phys. Rev. C 93, 065203 (2016)

39. B. Wang, L. Meng, S.L. Zhu, Phys. Rev. D 101, 034018 (2020)

40. M.A. Shifman, A.I. Vainshtein, V.I. Zakharov, Nucl. Phys. B 147, 385 (1979)

41. L.J. Reinders, H. Rubinstein, S. Yazaki, Phys. Rept. 127, 1 (1985)

42. K.C. Yang, W.Y.P. Hwang, E.M. Henley, L.S. Kisslinger, Phys. Rev. D 47, 3001 (1993)

43. J.R. Ellis, E. Gardi, M. Karliner, M.A. Samuel, Phys. Rev. D 54, 6986 (1996)

44. M. Eidemuller, M. Jamin, Phys. Lett. B 498, 203 (2001)

45. S. Narison, Camb. Monogr. Part. Phys. Nucl. Phys. Cosmol. 17, 1 (2002)

46. V. Gimenez, V. Lubicz, F. Mescia, V. Porretti, J. Reyes, Eur. Phys. J. C 41, 535 (2005)

47. M. Jamin, Phys. Lett. B 538, 71 (2002)

48. B.L. Ioffe, K.N. Zyablyuk, Eur. Phys. J. C 27, 229 (2003)

49. A.A. Ovchinnikov, A.A. Pivovarov, Sov. J. Nucl. Phys. 48, 721 (1988) [Yad. Fiz. 48, 1135 (1988)]

50. P. Colangelo, A. Khodjamirian, At the Frontier of Particle Physics/Handbook of $Q C D$, vol. 3 (World Scientific, Singapore, 2001) 